\title{
SELETIVIDADE DOS HERBICIDAS DICLOSULAM, FLUMETSULAM E CLORANSULAM EM DIVERSAS CULTIVARES DE SOJA
}

\author{
André Luiz Melhorança ${ }^{1}$ \\ ' Engenheiro Agrônomo, Dr., Pesquisador. Embrapa Agropecuária Oeste. Caixa Postal 661. Dourados, MS 79804-970 andre@cpao.embrapa.br
}

\begin{abstract}
RESUMO
A seletividade é a capacidade que um herbicida tem de controlar as plantas daninhas que se encontram em uma cultura sem reduzir a produtividade e a qualidade do produto final. Não pode ser determinada apenas pela verificação ou não de sintomas de fitotoxicidade, pois já são conhecidos exemplos de herbicidas que podem reduzir a produtividade das culturas sem produzir efeitos visualmente detectáveis e também de herbicidas que provocam injúrias bastante acentuadas, mas que permitem às culturas manifestar plenamente seus potenciais produtivos. Este trabalho teve como objetivo avaliar a seletividade dos herbicidas diclosulam, flumetsulam e cloransulam nas cultivares de soja BR-16, BR-9112410, Embrapa 64 (Ponta Porã), BR-37, BR-9113306, Lambari, Cambará, Embrapa-61, Conquista e Embrapa 65 (Itapoty). O experimento foi conduzido na área experimental da Embrapa Agropecuária Oeste, município de Dourados, MS, durante a safra de 1998/99. O ensaio foi delineado em blocos ao acaso com parcelas subdivididas, com quatro repetições. As parcelas foram os herbicidas e as subparcelas as cultivares. Realizaram-se avaliações de fitotoxicidade aos 14, 21 e 30 dias após aplicação dos tratamentos empregando-se o método de avaliação visual através de uma escala de 0 a 100, onde: $0=$ nenhuma injúria na planta e $100=$ morte total da planta. Avaliou-se a altura de planta, o número de plantas por metro e a produção de grão. A análise dos resultados permitiu concluir que o herbicida diclosulam na dose de $35 \mathrm{~g} / \mathrm{ha}$ e as misturas de diclosulam+flumetsulam nas doses de 20+94 g/ha, cloransulam+lactofen nas doses de $40+120 \mathrm{~g} / \mathrm{ha}$ e cloransulam+imazethapyr nas doses de 30+50 g/ha não afetaram a altura e o número de plantas por metro linear e o rendimento de grãos, mostrando serem altamente seletivos para as cultivares de soja BR-16, BR-9112410, Embrapa 64 (Ponta Porã), BR-37, BR-9113306, Lambari, Cambará, Embrapa-61, Conquista e Embrapa 65 (Itapoty).
\end{abstract}

Palavras-chave: cultivares, fitotoxicidade, Glycine max.

\section{ABSTRACT \\ Selectivity of herbicides diclosulam, flumetsulam, and cloransulam in several soybean cultivars}

Selectivity is the capacity of herbicides to control weeds in a crop without reducing the productivity and quality of the final product. It cannot be determined only by the verification of presence or not of toxicity symptoms, because there are already well-known herbicides that can reduce the productivity of crops without producing visual effects and also herbicides that provoke quite accentuated toxicity, but that allow the crop to manifest its productive potentials fully. This work has the objective to evaluate the selectivity of diclosulam, flumetsulam and cloransulam in soybean BR-16, BR-9112410, Embrapa 64 (Ponta Porã), BR-37, BR-9113306, Lambari, Cambará, Embrapa-61, Conquista and Embrapa 65 (Itapoty) cultivars. The experiment was carried out in the experimental area of Embrapa Agropecuária Oeste, in Dourados, MS, during 1998/99. The experimental design was a split plot, with four replications. The main plots were the herbicides and the subplots were the cultivars. Plant height, number of plants by meter and grain production were evaluated. Diclosulam at $35 \mathrm{~g} / \mathrm{ha}$ and mixtures of diclosulam+flumetsulam at the rates of $20+94 \mathrm{~g} / \mathrm{ha}$, cloransulam+lactofen at 40+120 g/ha and cloransulam+imazethapyr at 30+50 g/ha did not affect the height and 
number of plants by linear meter and the production of grains, showing to be highly selectives to soyben BR-16, BR-9112410, Embrapa 64 (Ponta Porã), BR-37, BR-9113306, Lambari, Cambará, Embrapa-61, Conquista and Embrapa 65 (Itapoty) cultivars.

Key words: phytotoxicity, Glycine max.

\section{INTRODUÇÃO}

A seletividade, segundo Velini et al. (1992), é a capacidade de um determinado herbicida eliminar plantas daninhas que se encontram em uma cultura sem reduzir a produtividade e a qualidade do produto final obtido; não pode ser determinada apenas pela verificação ou não de sintomas de fitotoxicidade, pois já são conhecidos exemplos de herbicidas que podem reduzir a produtividade das culturas sem produzir efeitos visualmente detectáveis e também de herbicidas que provocam injúrias bastante acentuadas, mas que permitem às mesmas manifestar plenamente seus potenciais produtivos. Estas informações são corroboradas por Lorenzi et al. (1994) quando dizem que níveis significativos de injúrias podem não causar redução de produtividade.

Conhecer adequadamente um herbicida corresponde à precisa determinação do conjunto de condições (incluindo doses, tipos de solo e vários outros fatores) em que o mesmo pode ser utilizado com a eficiência e a seletividade necessárias.

Cruz \& Gurgel (1983) verificaram que nos tratamentos com hexazinone os sintomas de fitotoxicidade desapareceram sem que ocorresse interferência na produção.

Existem trabalhos mostrando que apesar de ocorrer fitotoxicidade as plantas podem se recuperar e outros demonstrando o contrário, indicando que a expressão da fitotoxicidade por herbicida pode variar em função da variedade e do herbicida utilizado (Vitoria Filho \& Camargo, 1980; Velini et al., 1993a). O comportamento diferenciado das variedades frente à aplicação dos herbicidas tem sido ressaltado por diversos autores, entre eles Velini et al. (1993b) e, de acordo com Peixoto (1991), os processos de melhoramento das variedades têm contribuído para eliminar a suscetibilidade das plantas aos efeitos fitotóxicos dos herbicidas.

Este trabalho teve como objetivo avaliar a seletividade dos herbicidas diclosulam, flumetsulam e cloransulam nas cultivares de soja BR-16, BR-91 12410, Embrapa 64 (Ponta Porã), BR-37, BR-9113306, Lambari, Cambará, Embrapa-61, Conquista e Embrapa 65 (Itapoty).

\section{MATERIAL E MÉTODOS}

O experimento foi conduzido na área experimental da Embrapa Agropecuária Oeste, município de Dourados, MS, de acordo com normas preconizadas da Sociedade Brasileira da Ciências das Plantas Daninhas (1995), durante a safra de
1998/99. A semeadura e aplicação dos tratamentos em préemergência ocorreram em 12.12.98. Os tratamentos em pósemergência foram realizados em 05/01/99.

As cultivares de soja utilizadas foram BR-16, BR9112410, Embrapa 64 (Ponta Porã), BR-37, BR-9113306, Lambari, Cambará, Embrapa-61, Conquista e Embrapa 65 (Itapoty).

Foram realizadas as práticas culturais recomendadas para a região de acordo com Embrapa (1996).

O solo da área experimental apresentava as seguintes características de fertilidade e textura:

\begin{tabular}{l|ccc|cc|cccc}
\hline $\mathbf{p H}$ & \multicolumn{2}{|c|}{ Meq/100 de solo $(\mathrm{ml})$} & \multicolumn{2}{|c|}{$\mathrm{ppm}$} & \multicolumn{4}{c}{$\%$} \\
\cline { 2 - 10 } $\mathrm{H}_{2} \mathrm{O}$ & $\mathrm{Al}$ & $\mathrm{Ca}$ & $\mathbf{M g}$ & $\mathbf{P}$ & $\mathbf{K}$ & Areia & Argila & Silte & MO \\
\hline 5,8 & 0,0 & 3,1 & 1,7 & 3,8 & 200 & 13 & 71 & 15 & 2,4 \\
\hline
\end{tabular}

Os herbicidas utilizados foram:

a) diclosulam, com concentração de $840 \mathrm{~g} / \mathrm{kg}$, pertencente ao grupo químico das triazolopirimidina sulfonanilidas, formulação WG, tendo como nome comercial Spider;

b) flumetsulam, concentração de $120 \mathrm{~g} / \mathrm{l}$, herbicida do grupo químico das sulfonamidas, formulado como solução concentrada, nome comercial Scorpion;

c) cloransulam, concentração de $840 \mathrm{~g} / \mathrm{kg}$, grupo químico das triazolopirimidina sulfonanilidas, formulado como WG e nome comercial Pacto;

d) lactofen, concentração de $240 \mathrm{~g} / \mathrm{l}$, pertencente ao grupo químico dos difenil-éteres, formulado como concentrado emulsionável, com nome comercial de Cobra e

e) imazethapyr, $100 \mathrm{~g} / 1$, grupo químico da imidazolinonas, formulado como solução aquosa concentrada, nome comercial Pivot. As doses utilizadas no experimento são apresentadas na Tabela 1.

Os tratamentos foram aplicados com pulverizador costal propelido a $\mathrm{CO}_{2}$, equipado com barra de $1,5 \mathrm{~m}$ contendo quatro bicos leque 110.02 com pressão de $32 \mathrm{lb}$./pol. ${ }^{2}$, proporcionando uma vazão de 200 1/ha. As condições ambientais no momento da aplicação dos tratamentos de pré-emergência eram de tempo parcialmente nublado (25\%), temperatura do ar de $25^{\circ} \mathrm{C}$, umidade relativa de $72 \%$ e velocidade de vento de 0 a 1 $\mathrm{km} / \mathrm{h}$. Em pós-emergência o tempo apresentava-se limpo, temperatura do ar de $23^{\circ} \mathrm{C}$, umidade de $68 \%$ e velocidade de vento de 2 a $3 \mathrm{~km} / \mathrm{h}$.

O experimento foi delineado em blocos ao acaso com parcelas subdivididas com quatro repetições. As parcelas foram os herbicidas e as subparcelas, as cultivares As subparcelas mediam 4 × $5 \mathrm{~m}$. Os dados coletados foram submetidos à análise de variância através do teste " $F$ " e para 
comparação de médias adotou-se o teste de Tukey ao nível de 5\% de probabilidade (Pimentel Gomes, 1984 e 1985).

Realizaram-se avaliações de fitotoxicidade aos 14,21 e 30 dias após tratamento (DAT) empregando-se o método de avaliação visual através de uma escala de 0 a 100, onde: $0=$ nenhuma injúria na planta e $100=$ morte total da planta. Avaliou-se, também, a altura das plantas, a densidade e a produtividade da cultura.
As precipitações ocorridas no período de instalação do experimento foram: em 04/12/98 - $30 \mathrm{~mm}, 05 / 12 / 99$ - $35 \mathrm{~mm}$, $10 / 12 / 98$ - $15 \mathrm{~mm}, 11 / 12 / 98-1 \mathrm{~mm}, 15 / 12 / 98-1 \mathrm{~mm}, 16 / 12 / 98-$ $3 \mathrm{~mm}, 17 / 12 / 98-21 \mathrm{~mm}, 22 / 12 / 98-37 \mathrm{~mm}, 23 / 12 / 98-1 \mathrm{~mm}, 29 /$ $12 / 98-76 \mathrm{~mm}, 29 / 12 / 98-1 \mathrm{~mm}, 02 / 01 / 99$ - 3 mm, 03/01/99- 17 $\mathrm{mm}, 04 / 01 / 99-7 \mathrm{~mm}, 05 / 01 / 99-48 \mathrm{~mm}, 13 / 01 / 99-6 \mathrm{~mm}, 15 / 01 /$ $99-5 \mathrm{~mm}, 27 / 01 / 99-5 \mathrm{~mm}, 28 / 01 / 99-36 \mathrm{~mm}, 29 / 01 / 99-4 \mathrm{~mm}$, $31 / 01 / 99-21 \mathrm{~mm}$.

Tabela 1. Concentração e doses dos herbicidas utilizados no experimento. Embrapa Agropecuária Oeste, Dourados, MS, 1999.

\begin{tabular}{lccc}
\hline \multicolumn{1}{c}{ Tratamentos } & Concentração & \multicolumn{2}{c}{ Doses } \\
\cline { 3 - 4 } & (g/kg ou I) & g ou ml p.c./ha & g i.a./ha \\
\hline 1. Diclosulam & 840 & 41,67 & 35 \\
2. Diclosulam+flumetsulam & $840+120$ & $23,80+783,3$ & $20+94$ \\
3. Cloransulam+lactofen & $840+240$ & $47,61+500$ & $40+120$ \\
4. Cloransulam+imazethapyr & $840+100$ & $35,71+500$ & $30+50$ \\
5. Testemunha capinada & - & - & - \\
\hline
\end{tabular}

\section{RESULTADOS E DISCUSSÃO}

Na Tabela 2 são apresentados as médias de altura e o número de plantas por metro linear, percentagem de fitotoxicidade aos 14 e 21 DAT e rendimento de grãos em $\mathrm{kg} / \mathrm{ha}$. Os tratamentos (herbicidas) não afetaram significativamente o número e a altura de plantas e o rendimento de grãos, que apresentaram resultados estatisticamente semelhantes à testemunha capinada. Quanto à fitotoxicidade verifica-se que aos 14 DAT cloransulam + lactofen apresentou o maior sintoma $(20,1 \%)$, sendo estatisticamente semelhante a cloransulam + imazethapyr $(18,3 \%)$ e superior aos demais tratamentos. Aos 21 DAT houve uma sensível redução nos sintomas onde todos os tratamentos foram semelhantes entre si e superiores à testemunha capinada. Aos 30 DAT registrou-se ausência de fitotoxicidade para todos os tratamentos.

A ocorrência de fitotoxicidade aos 14 e 21 DAT não foi suficiente para causar redução no rendimento de grãos, que foi semelhante à testemunha capinada. Este resultado corrobora com Velini et al. (1992) e Lorenzi (1994) quando afirmam que níveis significativos de injúrias podem não causar redução de produtividade.
Quanto aos subtratamento, ou seja, as cultivares, observa-se que estas apresentaram diferenças significativas entre si quanto ao número de plantas, altura e rendimento de grãos, destacando a BR-37, Lambari e Cambará que apresentaram estande inferior ao das demais cultivares. Quanto à altura de planta, a cv. Conquista foi superior às demais. As melhores produções foram obtidas pela Conquista, Embrapa 65 (Itapoty), BR-16, BR 112410, Embrapa 64 (Ponta Porã) e BR-37, que foram semelhantes entre si. Quanto aos sintomas de fitotoxicidade aos 14 e 21 DAT todas as cultivares mostraram sintomas semelhantes entre si. A interação entre herbicida x cultivares não foi significativa para nenhum dos parâmetros avaliados. Este resultado, de certa forma, discorda de Vitória Filho \& Camargo (1980) e Velini et al. (1993a) quando afirmam que a expressão da fitotoxicidade por herbicida pode variar em função da variedade.

A análise geral dos resultados mostra que os herbicida diclosulam, diclosulam + flumetsulam, cloransulam + lactofen e cloransulam + imazethapyr nas doses testadas foram altamente seletivos para as cultivares BR-16, BR-9112410, Embrapa 64 (Ponta Porã), BR-37, BR-9113306, Lambari, Cambará, Embrapa-61, Conquista e Embrapa 65 (Itapoty). 
Tabela 2. Médias do número de plantas por metro linear, altura de planta, fitotoxicidade aos 15 e 30 dias após aplicação dos tratamentos (DAT) e rendimento de grãos. Embrapa Agropecuária Oeste, Dourados, MS, 1999.

\begin{tabular}{|c|c|c|c|c|c|}
\hline \multirow{2}{*}{ Tratamento/Subtratamento } & \multirow{2}{*}{$\begin{array}{l}\text { Número de } \\
\text { plantas/m }\end{array}$} & \multirow{2}{*}{$\begin{array}{l}\text { Altura } \\
(\mathbf{c m})\end{array}$} & \multicolumn{2}{|c|}{ Fitotocicidade (\%) } & \multirow{2}{*}{$\begin{array}{c}\text { Rendimento } \\
\text { de grãos } \\
\text { (kg/ha) }\end{array}$} \\
\hline & & & 14 DAT & 21 DAT & \\
\hline 1. Diclosulam & $10,1 \mathrm{a}$ & $72,9 \mathrm{a}$ & $15,2 \mathrm{c}$ & $5,1 \mathrm{a}$ & 2492 a \\
\hline 2. Diclosulam + flumetsulam & $9,8 \mathrm{a}$ & $73,9 \mathrm{a}$ & $17,4 \mathrm{bc}$ & $5.3 \mathrm{a}$ & $2380 \mathrm{a}$ \\
\hline 3. Cloransulam + lactofen & $10,1 \mathrm{a}$ & $74,1 \mathrm{a}$ & $20,1 \mathrm{a}$ & $4,6 \mathrm{a}$ & $2357 \mathrm{a}$ \\
\hline 4. Cloransulam + imazethapyr & $9,6 \mathrm{a}$ & $74,0 \mathrm{a}$ & $18,3 \mathrm{ab}$ & $5,0 \mathrm{a}$ & 2368 a \\
\hline 5. Testemunha capinada & $9,8 \mathrm{a}$ & $74,3 \mathrm{a}$ & $00,0 \mathrm{~d}$ & $0,0 \mathrm{~b}$ & 2171 a \\
\hline 1. BR-16 & $12,2 \mathrm{a}$ & 66,2 ef & $17,4 \mathrm{a}$ & $5,1 \mathrm{a}$ & 2772 a \\
\hline 2. BR-9112410 & $11,4 \mathrm{ab}$ & $63,6 \mathrm{f}$ & $17,3 \mathrm{a}$ & $5,2 \mathrm{a}$ & $2420 \mathrm{abc}$ \\
\hline 3. Embrapa 64 (Ponta Porã) & $10,4 \mathrm{abc}$ & 70,0 de & $17,0 \mathrm{a}$ & $5,0 \mathrm{a}$ & $2514 \mathrm{ab}$ \\
\hline 4. $\mathrm{BR}-37$ & $11,4 \mathrm{ab}$ & 67,3 ef & $17,3 \mathrm{a}$ & $5.1 \mathrm{a}$ & $2480 \mathrm{ab}$ \\
\hline 5. BR-9113306 & $7,8 \mathrm{de}$ & $50,9 \mathrm{~g}$ & $17,1 \mathrm{a}$ & $5,1 \mathrm{a}$ & $1985 \mathrm{~cd}$ \\
\hline 6. Lambari & $7,7 \mathrm{e}$ & $74,2 \mathrm{~d}$ & $17,4 \mathrm{a}$ & $5,0 \mathrm{a}$ & $1917 \mathrm{~d}$ \\
\hline 7. Cambará & 8,9 cde & $87,0 \mathrm{~b}$ & $17,0 \mathrm{a}$ & $5,0 \mathrm{a}$ & $1951 \mathrm{~d}$ \\
\hline 8. Embrapa 61 & 9,6 bcd & $83,3 \mathrm{bc}$ & $17,1 \mathrm{a}$ & $5,1 \mathrm{a}$ & 2267 bcd \\
\hline 9. Conquista & $10,0 \mathrm{bc}$ & $94,9 \mathrm{a}$ & $16,9 \mathrm{a}$ & $5,0 \mathrm{a}$ & $2554 \mathrm{ab}$ \\
\hline 10. Embrapa 65 (Itapoty) & $9,7 \mathrm{bc}$ & $81,2 \mathrm{c}$ & $17,1 \mathrm{a}$ & $5,0 \mathrm{a}$ & $2678 \mathrm{ab}$ \\
\hline F (Tratamento) & $0,52 \mathrm{~ns}$ & $0,28 \mathrm{~ns}$ & $12,8 * *$ & $246^{* *}$ & $2,18 \mathrm{~ns}$ \\
\hline F (Subtratamento) & $13,79 * *$ & $124,54 * *$ & $0,61 \mathrm{~ns}$ & $0,43 \mathrm{~ns}$ & $9,83 * *$ \\
\hline F (Trat. x Subtrat.) & $1,20 \mathrm{~ns}$ & $0,51 \mathrm{~ns}$ & $0,42 \mathrm{~ns}$ & $0,58 \mathrm{~ns}$ & $0,58 \mathrm{~ns}$ \\
\hline CV (\%) Tratamento & 21,0 & 9,2 & 1,5 & 1,3 & 21,0 \\
\hline CV (\%) Subtratamento & 18,3 & 7,0 & 2,5 & 1,8 & 18,8 \\
\hline
\end{tabular}

Médias seguidas de mesma letra não diferem significativamente pelo teste de Tukey ao nível de $5 \%$ de probabilidade.

\section{LITERATURA CITADA}

CRUZ, L.S.P.; GURGEL, M.N. do A. Efeitos de hexazinone e diuron, e suas misturas, no controle de capim-colchão (Digitaria sanguinalis) (L.) Scop) em cana-de-açúcar (Saccharum spp.). Planta Daninha, Campinas, v.6, n.1, p.15-20, jun. 1983.

EMBRAPA. Centro de Pesquisa Agropecuária do Oeste (Dourados, MS). Soja: recomendações técnicas para Mato Grosso do Sul e Mato Grosso. Dourados, 1996. 157p. (EMBRAPA-CPAO. Circular Técnica, 3).

LORENZI, H.J.; BRUNELLI NETO, V.; OLIVEIRA, J.E. Estudo do efeito do herbicida oxifluorfen, aplicado em pré-emergência, sobre o crescimento e produtividade da canade-açúcar (Saccharum officinarum) cv. SP 71-6163. STAB, Piracicaba, v.12, n.4, p.25-26, 1994.

PEIXOTO, A.B. Tratos culturais químicos. STAB, Piracicaba, v.9, n.3, p.18-21, 1991.

PIMENTEL GOMES, F.A. A estatística moderna na pesquisa agropecuária. Piracicaba: POTAFÓS, 1984. 160p.

PIMENTEL GOMES, F.A. Curso de estatística experimental. 11.ed. Piracicaba: Nobel, 1985. 466p.

SOCIEDADE BRASILEIRA DA CIÊNCIA DAS PLANTAS DANINHAS (Londrina, PR). Procedimentos para instalação, avaliação e análise de experimentos com herbicidas. Londrina, 1995. 42p.
VELINI, E.D.; FREDERICO, L.A.M.; MORELLI, J.L.; MARUBAUYASHI, O.M. Avaliação dos efeitos do herbicida clomazone, aplicado em pós-emergência inicial, sobre o crescimento e produtividade de soqueira de cana de açúcar (Saccharum officinarum cv. SP 71-1406). STAB, Piracicaba, v.10, n.4, p.13-16, 1992.

VELINI, E.D.; FREDERICO, L.A.M.; MORELLI, J.L.; MARUBAUYASHI, O.M. Avaliação dos efeitos do herbicida clomazone, aplicado em pós-emergência inicial, sobre o crescimento e produtividade de soqueira de cana de açúcar (Saccharum officinarum cv. SP 71-1406). STAB, Piracicaba, v.12, n.2, p.30-36, 1993a.

VELINI, E.D.; FREDERICO, L.A.M.; MORELLI, J.L.; MARUBAUYASHI, O.M.; KOJIMA, K. Avaliação dos efeitos do herbicida clomazone, aplicado em pós-emergência sobre o crescimento e produtividade de soqueira de nove cultivares de cana-de-açúcar. In: CONGRESSO NACIONAL DA STAB, 5., 1993, Águas de São Pedro, SP. Programa e resumos ... [S.1.: s.n., 1993b].

VICTORIA FILHO, R.; CAMARGO, P.N. de. Efeitos de herbicidas nos teores de macronutrientes e nas características tecnológicas da cana-de-açúcar (Saccharum spp.). I - Misturas de herbicidas em pós-emergência. Planta Daninha, Campinas, v.3, n.2, p.96-107, dez. 1980. 